\title{
Tonotopic reorganization and spontaneous firing in inferior colliculus during both short and long recovery periods after noise overexposure
}

\author{
Feng Wang ${ }^{1 \dagger}$, Li Zuo ${ }^{2,3 \dagger}$, Bo Hong ${ }^{4}$, Dongyi Han ${ }^{5}$, Ethan M Range ${ }^{3}$, Lingyun Zhao ${ }^{4}$, Yanan Sui ${ }^{4}$, Weiwei Guo ${ }^{5}$ \\ and Liangfa Liü, ${ }^{5,6^{*}}$
}

\begin{abstract}
Background: Noise induced injury of the cochlea causes shifts in activation thresholds and changes of frequency response in the inferior colliculus (IC). Noise overexposure also induces pathological changes in the cochlea, and is highly correlated to hearing loss. However, the underlying mechanism has not been fully elucidated. In this study, we hypothesized that overexposure to noise induces substantial electrophysiological changes in the IC of guinea pigs.

Results: During the noise exposure experiment, the animals were undergoing a bilateral exposure to noise. Additionally, various techniques were employed including confocal microscopy for the detection of cochlea hair cells and single neuron recording for spontaneous firing activity measurement. There were alterations among three types of frequency response area (FRA) from sound pressure levels, including $\mathrm{V}-, \mathrm{M}$-, and $\mathrm{N}$-types. Our results indicate that overexposure to noise generates different patterns in the FRAs. Following a short recovery (one day after the noise treatment), the percentage of V-type FRAs considerably decreased, whereas the percentage of M-types increased. This was often caused by a notch in the frequency response that occurred at $4 \mathrm{kHz}$ (noise frequency). Following a long recovery from noise exposure (11-21 days), the percentage of $\mathrm{V}$-types resumed to a normal level, but the portion of M-types remained high. Interestingly, the spontaneous firing in the IC was enhanced in both short and long recovery groups.
\end{abstract}

Conclusion: Our data suggest that noise overexposure changes the pattern of the FRAs and stimulates spontaneous firing in the IC in a unique way, which may likely relate to the mechanism of tinnitus.

Keywords: Cochlea, Noise, Tinnitus, Hair cells, Inferior colliculus

\section{Background}

Tinnitus is a condition normally associated with hearing loss, and involves the perception of sound without the corresponding stimulus [1-3]. The reorganization of the frequency topographic map (FTM) has shown to be a direct outcome from hearing loss [4-7]. This could be associated with an increase of spontaneous firing in the brain auditory center $[8,9]$. It is accepted that such

\footnotetext{
* Correspondence: labrat304@gmail.com

${ }^{\dagger}$ Equal contributors

${ }^{5}$ Department of Otolaryngology-Head and Neck Surgery, Chinese PLA General Hospital, Beijing 100853, China

${ }^{6}$ Department of Otolaryngology, Beijing Friendship Hospital, Capital Medical University, Beijing 100050, China

Full list of author information is available at the end of the article
}

reorganization and spontaneous firing $[1,3,10]$ are likely involved in the mechanism of tinnitus.

Previous studies have determined that the auditory system contains a topographic representation of tone frequency. The inner hair cells of the cochlea are tonotopically organized, resulting in each inner hair cell having tonotopic connections, through its associated ganglion cells, to cells in the cochlear nucleus [11]. Interestingly, topographic plasticity in the adult central auditory system has been well documented using a wide variety of techniques that result in partial ablation within the periphery of the auditory system. These techniques include mechanical disruption of the organ of Corti $[5,6]$ and spiral

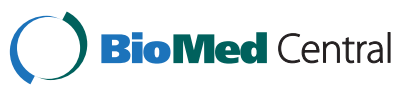

(c) 2013 Wang et al.; licensee BioMed Central Ltd. This is an Open Access article distributed under the terms of the Creative Commons Attribution License (http://creativecommons.org/licenses/by/2.0), which permits unrestricted use, distribution, and reproduction in any medium, provided the original work is properly cited. The Creative Commons Public Domain Dedication waiver (http://creativecommons.org/publicdomain/zero/1.0/) applies to the data made available in this article, unless otherwise stated. 
ganglion [7,12], administration of cochleotoxic drugs $[4,13,14]$, and exposure to high intensity sounds $[15,16]$.

The documented changes in the inferior colliculus (IC) included: expanded lesion-edge frequency representations, shifts in characteristic frequencies that cause the expanded lesion edge frequency representations within affected frequency-response areas (FRA) [7,12], reduced levels of sideband inhibition at the particular frequency causing hearing loss $[12,15,17]$, and reduced inhibition response from multi-units [4]. The changes within the IC occurred immediately after lesions developed and continued for several weeks to several months. For example, hearing loss greater than $10 \mathrm{~dB}$, induced by noise exposure, caused instantaneous changes in the primary auditory cortex [15].

At a single-unit level, tonotopic re-organization of the IC is related to a shift in the characteristic frequency (CF). The shift included a distinct staircase pattern [18] along the dorsoventral axis. Following noise exposure, the original stepwise frequency-depth function changed significantly. In some distortions, only two or three "steps" appeared. The V-type, which is most common in the IC $[7,12,19]$, translated to double peaks or multipeaks in the FRA. Non V-type FRAs included two or three frequency peaks [16]. The CF thresholds significantly increased with the time following noise exposure, partially accounting for the occurrence of tinnitus [15]. Furthermore, previous reports have shown that auditory neural hyperactivity appears in the auditory cortex, IC, and cochlear nucleus, occurring simultaneously with tinnitus $[1,20]$. Yet, the mechanisms of tinnitus, which are associated with hearing loss, have not been clearly identified in these models.

To understand these complex mechanisms associated with tinnitus, narrow-band noise was utilized to induce cochlear damage in guinea pigs. We have measured the FRAs, spontaneous firing of single units in the IC, and cochlear damage in a single cell level, following noise overexposure of one day (short recovery) and 11-21 days (long recovery), respectively. Our data provide a new perspective in understanding the mechanism of noise induced tinnitus.

\section{Methods}

All procedures were approved by the Department of Otolaryngology at The First Affiliated Hospital of Chinese PLA General Hospital and followed all required guidelines. Care and use of the animals in this study was also approved by the Institutional Animal Care and Use Committee of the Chinese PLA General Hospital. Nineteen healthy guinea pigs of both sexes with normal auricular reflex, weighing between $227 \mathrm{~g}$ and $525 \mathrm{~g}$ were used. The animals were randomly divided into three groups. Short recovery group (7 animals) was evaluated 24 hours after noise exposure and long recovery group was evaluated 11-21 days after exposure (6 animals). In the control group, six healthy animals were used. This timeframe (11-21 days) was consistent with previous studies, which showed that compound action potential (CAP) thresholds were fractionally recovered two weeks after noise-exposure $[3,21,22]$.

\section{ABR thresholds}

The auditory brainstem response (ABR) was also measured. Specifically, guinea pigs were anesthetized with xylazine $(0.1 \mathrm{mg} / \mathrm{kg})$ and ketamine $(30 \mathrm{mg} / \mathrm{kg})$. Subdermal electrodes were inserted at the vertex and pinna. A speaker was positioned directly above the mid-line of the animal's head at a height of $12 \mathrm{~cm}$. Tone-pips $(0.5-\mathrm{ms}$ on rise/fall time, at $30 / \mathrm{sec}$ ) at $2,4,8$, and $16 \mathrm{kHz}$ were used as sound stimuli. The response signal was amplified, filtered, and averaged using the Intelligent Hearing System software (ISEN Tech and Trading, Ltd. Beijing, China) [23].

\section{Conditions for noise exposure}

During this procedure, the animals were not anesthetized and thus were fully awake, undergoing a bilateral exposure to noise, which was produced from a signal machine (Model 33220A, Agilent Technologies, Inc., UK), intensified by a power amplifier (Model 2706, Brüel \& Kjær Sound \& Vibration Measurement A/S, Denmark), and transmitted to the surrounding speakers (Model D1002, Beijing First Radio Equipment Factory, Beijing, China). The acoustic overexposure stimulus was a $1 / 3$ octave narrow band noise (i.e. white noise filtered by $1 / 3$ octave band filter) at $4 \mathrm{kHz}$ and $120 \mathrm{~dB}$, measured by a sound level meter (Model 2209, Brüel and Kjær Sound and Vibration Measurement A/S, Denmark), and lasted 4 hours within a sound insulated room. The speakers were placed $4 \mathrm{~cm}$ in front of the head of an unrestrained guinea pig within small cells in a subdivided cage ( 1 animal/cell). Noise calibration, targeting SPL, was performed immediately before each exposure session. It should be noted that sound pressure levels varied by $<1 \mathrm{~dB}$ across the cages.

\section{Surgery procedure}

Before the surgery, a dosage of $0.7 \mathrm{ml} / 100 \mathrm{~g}$ urethane was injected intraperitoneally for anesthetic purposes. The level of anesthesia was monitored in accordance with breathing state and blink reflex during the experiment. If the blink reflex occurred, one third of the concentration of the first dose of urethane was added to maintain a state of unconsciousness. The animals were given a preoperative subcutaneous injection of a dosage of $0.05 \mathrm{ml} / 100 \mathrm{~g}$ atropine to reduce respiratory secretion and to prevent respiratory tract obstruction during the experiment. Oxygen was administered through a customized mask 
while conducting the experiment. The animal body temperature was maintained by an electric blanket (Model 050100C0001B, FHC Inc. Bowdoin, ME, USA). After the guinea pigs were anesthetized, they were placed in the stereotaxic apparatus. The scalp, temporal muscle, and connective tissue were removed and the skull was exposed. A $10 \mathrm{~cm}$ long flat head screw was fixed on the top of the skull using glue and reinforced by dental cement (Model: 200805, Dental Division in Shanghai Medical Equipment, Ltd., Shanghai, China). An area which is $2 \mathrm{~mm}$ behind lambda, and $2.5 \mathrm{~mm}$ to the right side of the midline, was marked as a circle center with a radius of $2.5 \mathrm{~mm}$. The skull included in the radius of the circle was removed by a micro-drill and the dura mater was removed.

\section{Generation of stimuli}

Digital signals generated with $200 \mathrm{kHz}$ sampling rate were used as the sound stimuli. After analog conversion (RX6; Tucker-Davis Technologies, USA) [24,25], the stimuli were applied to the anesthetized animals through the speakers (Electrostatic speaker 1, TDT Co., USA) which were placed next to each ear $20 \mathrm{~cm}$ from lambda in a free field. The rise/fall time of pure tone was $5 \mathrm{~ms}$ [17]. The stimulation sequences included two steps: 1) A sweep sequence was used for the determination of $\mathrm{CF}$ and minimum threshold of the IC neurons, followed by random sweep sequences including 561 pure tones ( $50 \mathrm{~ms}$ duration) at an interval of $250 \mathrm{~ms}$ between each tone pip; in this set-up, the frequency ranged from $1 \mathrm{kHz}$ to $60 \mathrm{kHz}$ with 5.9 octaves, and sound intensities were randomly ranged from 10 to $60 \mathrm{~dB}$ SPL with 11 linear steps on the intensity curves. 2) Repetitive stimulation sequences were used for determination of neuronal discharge patterns at CF $40 \mathrm{~dB}$ above threshold; this was repeated 100 times with a stimulation interval of $600 \mathrm{~ms}$ and duration of $200 \mathrm{~ms}$.

\section{Single neuron recording}

Extracellular electrophysiological recordings were performed in an anechoic chamber where the anesthetized guinea pigs were fixed in place with both ears in a natural unblocked state, which allowed all data obtained in the IC to be binaural. The speakers were placed next to each ear $20 \mathrm{~cm}$ from lambda. Single tungsten electrodes (Model 575300, A-M Systems, Sequim, WA, USA), with tip diameters of 50 microns, were controlled by micro-thrusters. Each electrode track through the IC was continued until a reduction in field potential amplitude was observed. The electrode was then immediately withdrawn and repositioned and a new track was started. The raw signal was sampled at $25 \mathrm{kHz}$, amplified (X 5000; A-M systems, Sequim, WA, USA), and filtered $(0.3 \sim 3 \mathrm{kHz}$; TuckerDavis Technologies, TDT Co., Alachua, FL, USA). The
FRA, CF and threshold at CF of a single IC neuron were determined audio-visually after a sweep sequence was used. Spontaneous activities of the IC neuron were identified during this recording process, following the criteria: firing rate $\geq 5$ spikes $/ \mathrm{s}$, duration $\geq 10 \mathrm{~s}$. Data were analyzed with OpenSorter software 2.5.0 (TuckerDavis Technologies, TDT Co., Alachua, FL, USA). The typical IC neurons with spontaneous activities were detected according to the FRAs and the post stimulus time histogram (PSTH) as described previously $[10,19]$.

\section{Nuclear DNA staining of cochlear hair cell}

DNA fluorescent dye Hoechst 33342 (Sigma, St. Louis, USA) was used for the nuclear staining of the Organ of Corti [26]. Hoechst was prepared as $0.04 \%$ (wt/vol) stock solution using distilled water and stored in the dark at $4^{\circ} \mathrm{C}$. For staining, the stock solution was diluted to $1: 200$ by phosphate buffered saline (PBS, Beijing Puboxin BioTech Ltd., Beijing, China). After signal recording in IC cells, the animal was sacrificed. The temporal bone was removed along with the opening of the oval window and round window of the ear. A solution of 4\% paraformaldehyde was repeatedly infused into the cochlea through both windows. Then the cochlea was quickly dissected from the surrounding bones, and the basilar membrane was isolated, being placed in Hoechst staining solution for 10 minutes in the dark at room temperature. The specimen was washed in the PBS and mounted using 50\% glycerol (Beijing Puboxin BioTech Ltd., Beijing, China) on a slide. The fluorescence excitation wavelength for Hoechst 33342 was $337 \mathrm{~nm}$, and the corresponding emission wavelength was $430 \mathrm{~nm}$. Imaging was performed using an Olympus confocal microscope (FluoView FV1000, Olympus China Ltd., Beijing, China), which can be used to count the number of hair cells in the randomly selected areas.

The total number of nuclei in either inner or outer hair cells was counted from three 1-mm sections in length located within the cochlea duct. For the control group, we used 12 cochleae from six guinea pigs. Similarly, for the long recovery group, 12 cochleae from six animals were also examined. The three sites were chosen at $14.5-15.5,10.5-11.5$, and $4.0-5.0 \mathrm{~mm}$ from the basal end of the basilar membrane, respectively. The nucleus condition was determined through visual inspection under microscopy.

\section{Statistical analysis}

All results were presented as mean \pm standard error (mean $\pm \mathrm{SE}$ ). Comparisons of constituent proportions among the three groups (FRA) were made using PASW Statistics 18.0 software and SPSS 13.0 software (SPSS Inc. IBM Co. Armonk, New York, USA). Comparisons between groups were performed through Student's t-Test, and $P<0.05$ was considered significant. 


\section{Results}

\section{ABR thresholds in noise exposure group}

Prior to noise exposure, the average auditory brainstem response (ABR) threshold was screened using 2, 4, 8, and $16 \mathrm{kHz}$. One day after noise overexposure, the ABR thresholds were raised at all the above frequencies compared to pre-exposure levels $(P<0.01$ from 13 animals). The specific data are listed in Figure 1.

\section{Nuclear changes of inner and outer hair cells of the cochlea following noise exposure}

During the long recovery after noise overexposure, we found significant portions of fragmentation or disappearance of the nuclei within both outer and inner hair cells of the cochlea, as compared to control; for inner hair cells, irregular shape and disorganized alignment of the nuclei were observed (Figure 2A-D). Furthermore, it appears that there are more considerable nuclear disruptions in the 1st and 2nd turn of the cochlea compared to the 3rd turn, suggesting more high frequency damages (in 1st and 2nd turns) than low frequency damage (3rd turn). It has been noted that Figure $2 \mathrm{~A}$ is a typical picture represented for 1st, 2nd, or 3rd turn in control cochlea (no NE), because in our confocal settings, there was no marked difference among the images taken from these cochlear sections. Thus, Figure 2A was used as a general image taken from the 1st to 3rd turns.

As shown in Figure 3, the number of cochlea nuclei in the after-noise exposure (NE) group was significantly reduced compared to pre-NE group (213.1 \pm 3.5 vs. $242.5 \pm 2.1$ for inner hair cells; $615.9 \pm 12.5$ vs. $720.9 \pm 12.5$ for outer hair cells; $n=12, P<0.05$ ).

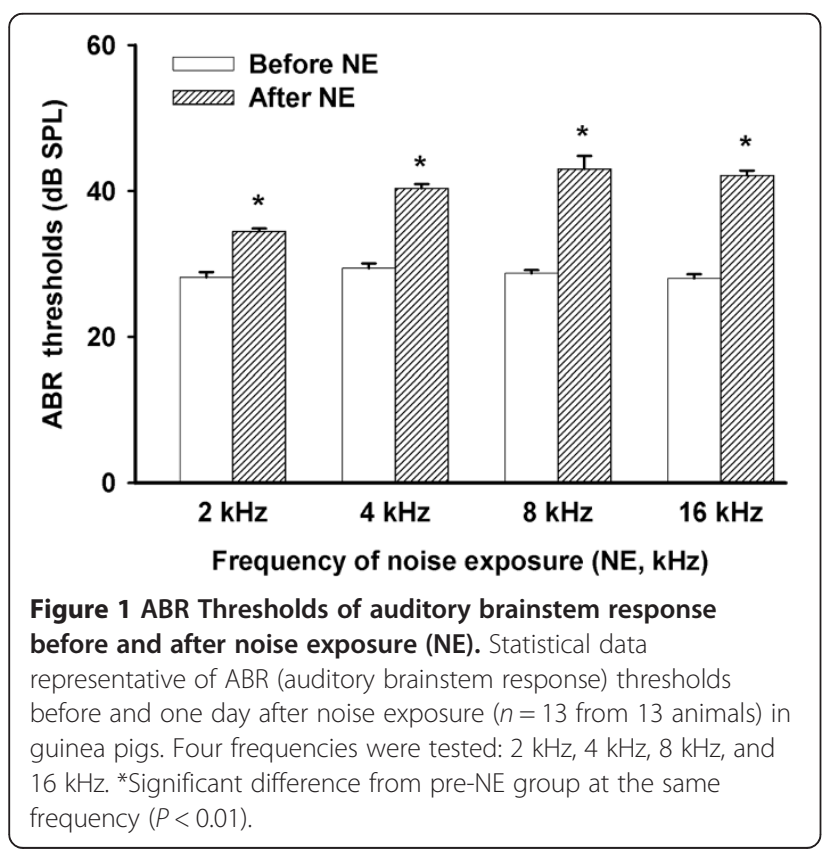

\section{Composition change of FRA}

The description of the FRA follows the methods of Hernandez et al. [19]. The FRA types of recorded neurons from all guinea pigs we used were similar to those previously reported [19]. The majority of FRAs have a single peak, the V-type, as defined by Hernandez et al. [19]. In order to compare the V-type to other types, the double peak or multi-peak type was defined as M-type, and the elongated narrow rectangular band was defined as N-type (Figure 4). As shown in Figure 5, during a short recovery period after noise overexposure, the percentage of V-type was significantly lower than the normal group $(61.1 \pm 2.4 \%$ vs. $86.6 \pm 4.7 \%, n=6-7, P<0.01)$. During the long recovery period, the percentage of $\mathrm{V}$-type shifted back towards normal $(76.6 \pm 7.8 \%$ vs. $86.6 \pm 4.7 \%, n=6$, n.s.). The percentage of $\mathrm{M}$-type is significantly increased during both short $(19.3 \pm 5.4 \%, n=7, P<0.05)$ and long recovery groups $(16.7 \pm 6.7 \%, n=6, P<0.05)$ compared to the normal group $(6.0 \pm 3.1 \%, n=6)$. However, the percentage of N-type remained constant in both short and long recovery periods (Figure 5).

\section{Changes of characteristic frequency (CF) over depth}

As shown in Figure 6A, the neuronal CF increased with the increasing depth of the tungsten electrode insertion along the dorsoventral axis of the IC in normal groups (114 neurons from 6 animals). Interestingly, we have observed that for the short recovery group, there is a noticeable gap at $4 \mathrm{kHz}$ (161 neurons from 7 animals, Figure 6B, $P<0.01$, neurons with $4 \mathrm{kHz}$ gap in short recovery group vs. neurons with $4 \mathrm{kHz}$ gap in normal group, post hoc contrast). However, this gap was partially reduced in the long recovery group (74 neurons from 6 animals, $P<0.01$, neurons with $4 \mathrm{kHz}$ gap in long recovery group $v s$. neurons with $4 \mathrm{kHz}$ gap in normal group; $P<0.05$ neurons with $4 \mathrm{kHz}$ gap in long recovery group $v s$. in neurons with $4 \mathrm{KHz}$ gap in short recovery group, post hoc contrast) (Figure 6C).

\section{Changes of IC neurons with spontaneous firing}

Representative FRA with typical spontaneous firing activity (A), corresponding spontaneous firing curve (B), and the percentage of neurons with spontaneous firing activity in different groups (C) are illustrated in Figure 7. Spontaneous activities in the neuron were identified following the criteria: firing rate $\geq 5$ spikes/s, duration longer than $10 \mathrm{~s}$. Thus, any firing rate lower than 5 spikes/s was regarded as the absence of spontaneous activities. Specifically, in Figure 7C, the percentage of IC neurons with spontaneous firing activity in the short recovery group was significantly increased compared to normal group $(31.6 \pm 3.6 \%$ vs. $15.3 \pm 3.0 \%, n=6-7, P<0.05)$. The percentage of IC neurons showing spontaneous activity in the long recovery group was also higher than normal 

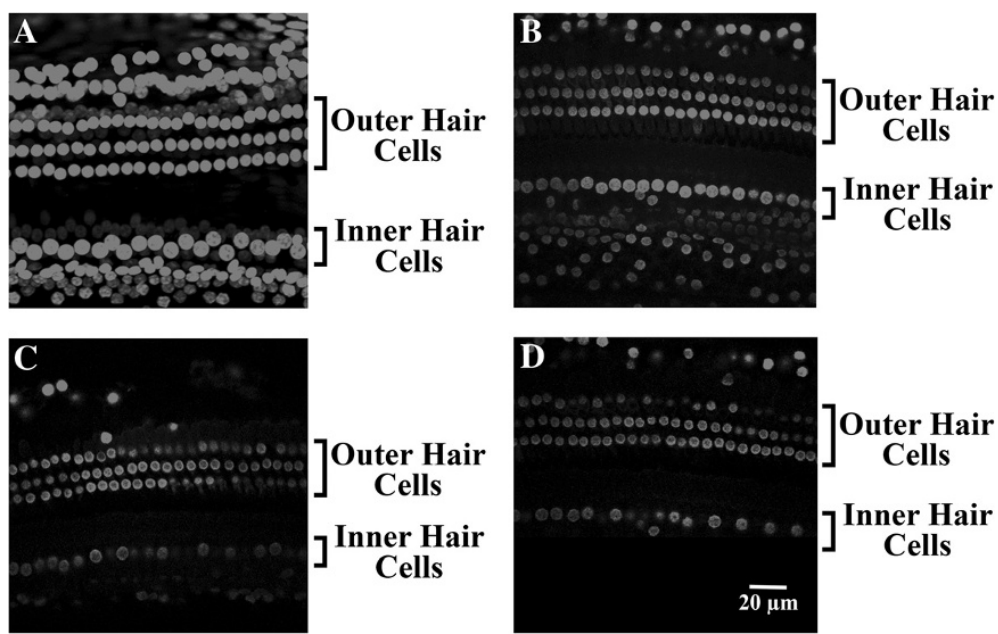

Figure 2 Representative confocal microscopy $(x 40)$ of fluorescence stained nuclei within inner and outer hair cells in the cochlea of guinea pigs, 11 days after noise exposure (120 dB, 4 hours). A: Normal levels are observed in the nuclei of inner and outer hair cells of the control subject. The nuclei were arranged normally and no disruption or fragmentations were found. $\mathbf{B}$ : The image location is in the third turn of the cochlea after noise exposure, where signs of fragmentation equally occurred throughout both inner and outer hair cells. C: In the second turn of the cochlea, after noise exposure, more disruption and fragmentation appears to occur in the inner and outer hair cell nuclei compared to the third turn of the cochlea. D: The first turn in the cochlea, after noise exposure, exhibited severe disruption and fragmentation compared to the second and third turns in the inner and outer hair cell nuclei.

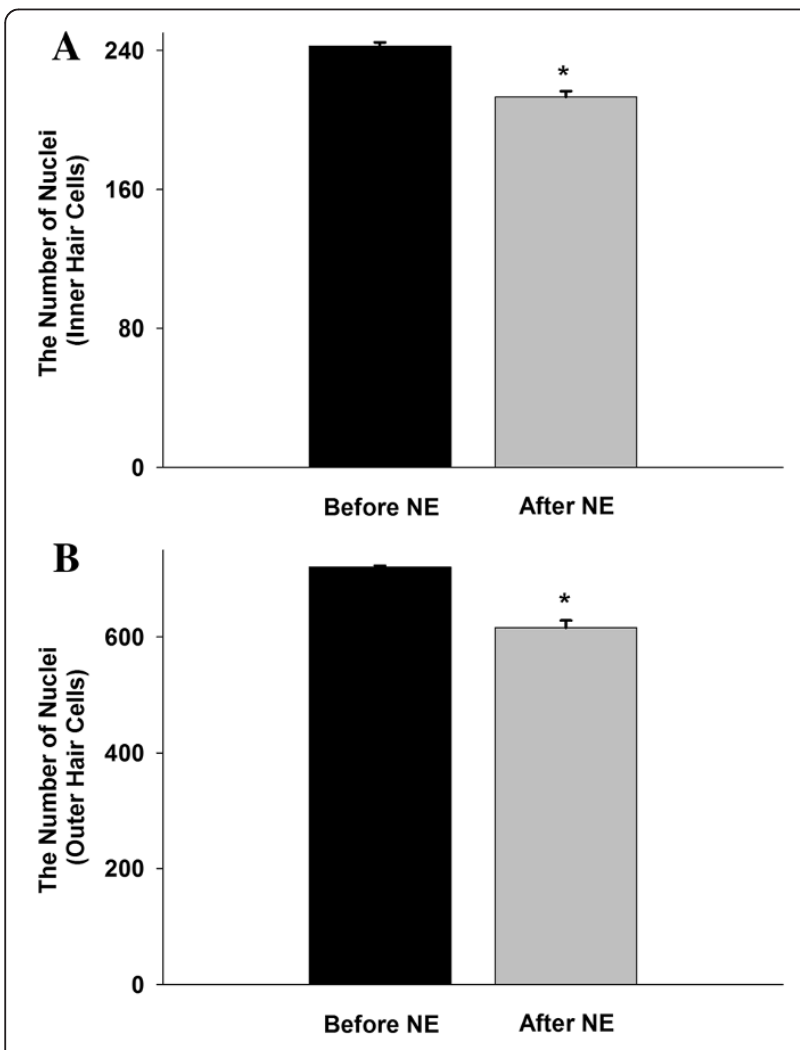

Figure 3 The number of nuclei in the cochlea in inner (A) and outer (B) hair cells. Before noise exposure (NE) vs. after NE $(n=12$ from 6 animals; $\left.{ }^{*} P<0.05\right)$. group $(40.5 \pm 13.8 \% \quad$ vs. $15.3 \pm 3.0 \%, \quad n=6, \quad P<0.05)$. There was no statistical difference between the short and long recovery groups.

\section{Discussion}

In this study, noise exposure caused two pathological consequences: one is FRA constituent proportions which were noticeably changed; the other is the IC cells which showed higher spontaneous firing activities. We also observed that average ABR thresholds at $4 \mathrm{kHz}, 8 \mathrm{kHz}$, and $16 \mathrm{kHz}$ were $\sim 10 \mathrm{~dB}$ higher in the acute noise exposure group. In addition, images of nucleus staining in the hair cells clearly showed extensive disruption and fragmentations in both inner and outer hair cells in the 1st and 2nd turns after NE. However, we are not clear whether the cells were undergoing inflammation or apoptosis. Thus, we only speculate that the cells could possibly be in these stages, which requires further studies.

Consistent with previous studies, narrow-band frequency noise, or pure tone could cause a diffused elevation of CAP thresholds in adjacent frequencies which are normally higher than the exposure frequency. This may be due to the basalward shift of damage to inner and outer hair cells located on the basilar membrane [2,27,28]. According to Seki's study, hearing loss of more than $10 \mathrm{~dB}$ can cause changes of auditory central CF maps in cats $[12,15,17]$. Moreover, in our study, the underlying causes of the histogram changes (Figure 5) may be complex. FRA constituent proportions' changes may be induced by elevation of ABR thresholds. After noise exposure, the neuronal output of the cochlea decreased proportionally along with 

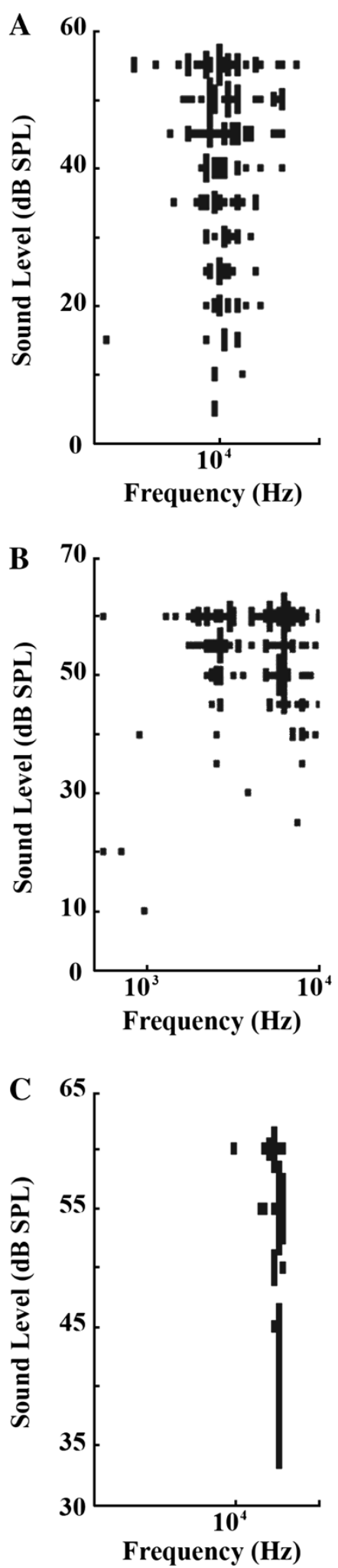

Figure 4 Representative graphs of frequency response patterns that are the basis of FRA types. (A) V-type, (B) M-type, (C) and $\mathrm{N}$-type FRAs created by testing neuronal responses through electrode measurement. Variables included sound frequency and intensity. the increased numbers of damaged inner hair cells [17]. Mechanistically, inner hair cells can accurately convert nano level ciliary beats, caused by sound waves, to electrical energy [29]. Outer hair cells can process the space-time coding through a conversion from mechanical energy to the electrical energy generated by the inner hair cells [29]. Therefore, damage to outer hair cells caused changes of space-time coding for output sound, resulting in a decreased cochlear signal output. Thus, the inhibition/excitation balance of IC neurons became disrupted and new sound space-time coding was reconstructed.

We have found that FRA constituent proportions noticeably changed after noise exposure, which is consistent with previous studies $[12,15,17]$. Specifically, the aforementioned change was most notable in the short recovery group regarding V- and M-type. The long recovery following noise exposure allowed for the continuous restructuring of FRA types back to normal levels, resulting in reduced differences among proportions of FRA types between normal and long recovery groups. This suggests that IC neurons show signs of self-repair during a prolonged time period. These changes in tuning curves are possibly correlated to the fundamental cause of tinnitus or hyperacusis (oversensitivity) [30], which will be the focus of further research.

In the function curves of $\mathrm{CF}$ and depth in our experiments, there is a marked gap at $4 \mathrm{kHz}$ measured in the short recovery group as shown in the CF vs. depth map. The reduced gap observed in the long recovery group implied the presence of self-repair processes 11-21 days following noise exposure. Although, when compared to chronic tinnitus, the period of $11-21$ day is relatively

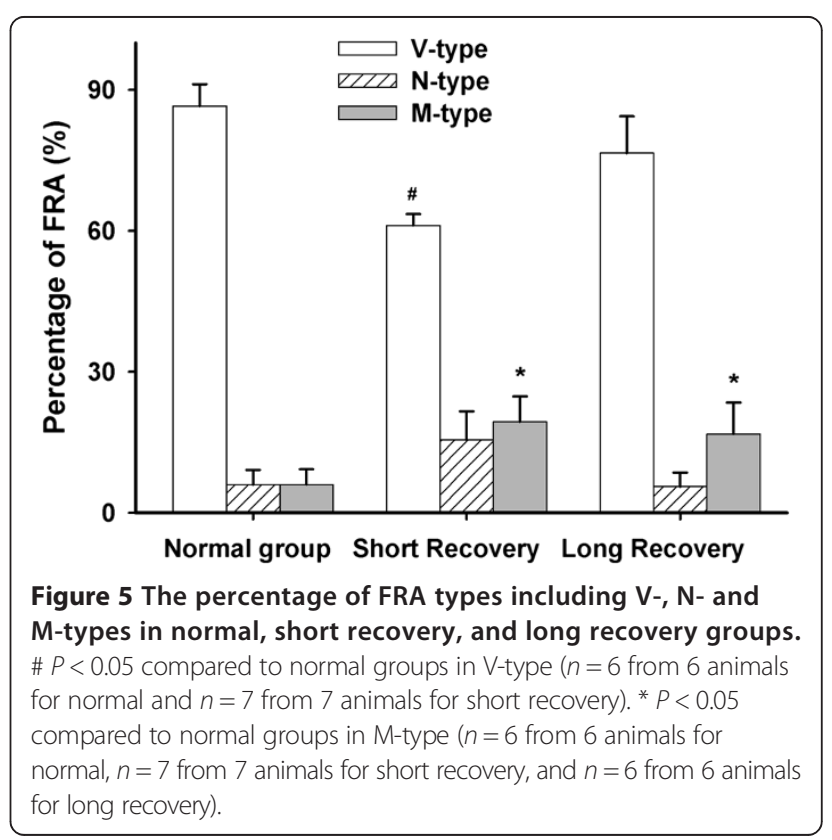


Figure 6 Function curves providing a correlation between CF (characteristic frequency) and protrusion depth within the IC. A: A normal distribution can be observed within the control CF map. CF and depth have a positive trend, resulting in higher frequencies the deeper the protrusions within the IC. B: Short recovery group shows more of a randomized pattern with dispersed responses above and below $4 \mathrm{kHz}$ creating a gap specifically at that frequency. C: Long recovery group exhibited the same type of gap at $4 \mathrm{kHz}$, but at a reduced level showing signs of recovery.

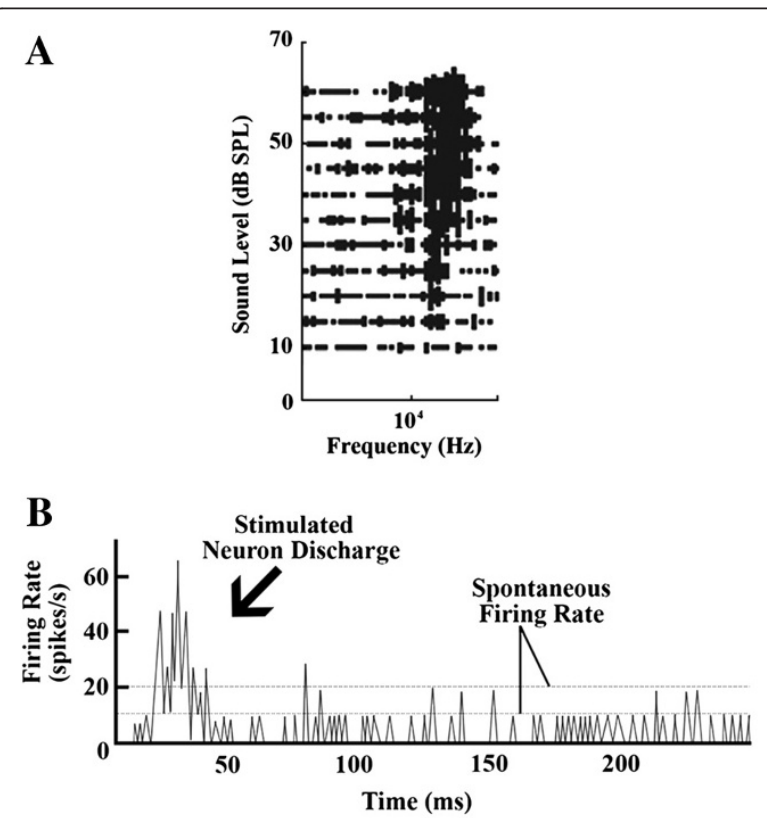

C

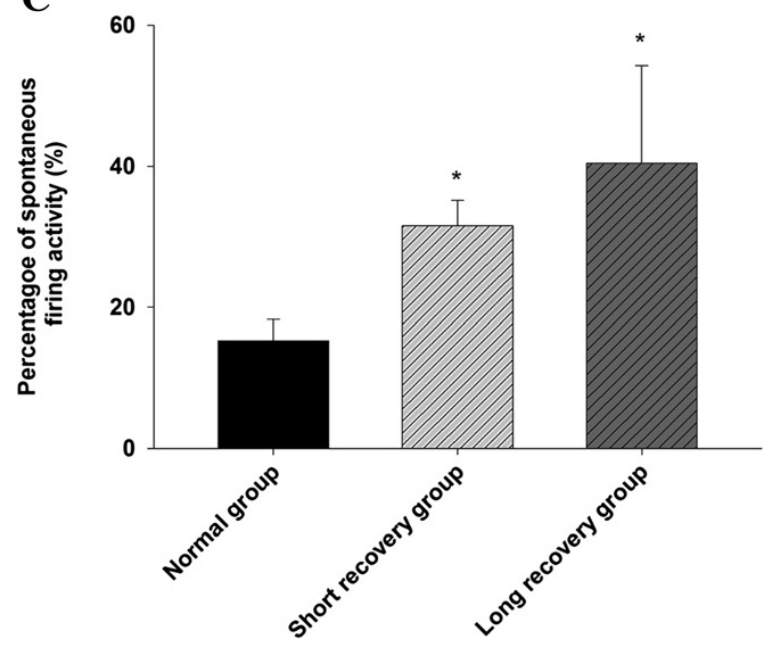

Figure 7 Spontaneous firing activities in IC neurons.

A: Representative FRA showing typical spontaneous firing. B: A typical spontaneous firing curve over time in an IC neuron. C: The percentage of neurons with spontaneous firing activity in normal (114 neurons from 6 animals), short recovery (161 neurons from 7 animals) and long recovery groups (74 neuron from 6 animals). *Significant difference from normal group $(P<0.05)$. 
short, it provides a sufficient timeframe to measure the pathological changes in hearing during this period. Note that the noise overexposure frequency was set at $4 \mathrm{kHz}$, which correlated with this gap. A former study showed that the maximal inhibitory frequency is normally lower than the major frequency at which tinnitus occurs [31]. Our research suggests that the noise of $4 \mathrm{kHz}$ caused a significant shift at 4, 8 and $16 \mathrm{kHz}$ in ABR thresholds.

Increased spontaneous firing rates and the reorganization of the tonotopic map closely follow sensory deafferentation. This also provides an objective signal for tinnitus when associated with hearing loss [32]. It has been reported that after tone-induced $(6 \mathrm{kHz})$ hearing loss in cats, increased synchrony was largely restricted to regions of the auditory cortex where reorganization of the tonotopic map occurred $(6-10 \mathrm{kHz})$ compared to non-reorganized regions [8].

In this study, we observed that the FTM reorganization was more noticeable in the short recovery group than the long recovery group. Both groups also had higher spontaneous firing activities than normal. The reorganization of the FTM is obvious in either recovery period when the increased spontaneous firing activity occurs, suggesting that the firing is a relatively dependent event with FTM reorganization. Interestingly, spontaneous rates are increased in animals with tinnitus [33]. Other research has shown that neural tinnitus can continue for life-long periods after recovery from noiseinduced damage, whereas CAP thresholds recover only partially weeks after noise-exposure $[3,21,29]$. Therefore, we speculate that these enhancements in spontaneous firing within the IC neurons relate to tinnitus models previously described [34]. The mechanism of increased spontaneous firing after noise exposure has not yet been fully understood. However, it is likely related to hyperactivity of the auditory nuclei in the brain stem, or a reduction in the normal suppressive activity of the central auditory cortex on peripheral auditory nerve activity as indicated in previous research [35]. It is possible that the disruption of inhibition/excitation balance in IC may arise from noise induced damage to both inner- and outer-hair cells, resulting in increased spontaneous activities in the IC neurons.

\section{Conclusion}

Our research has determined the response of FRAs following noise overexposure. IC neurons during both short and long recovery periods show increased spontaneous firing activity after noise overexposure, when compared to the normal group. In addition, we found that the spontaneous firing and FTM reorganization are two correlated events. Noise induced damage in both inner- and outer- hair cells should be related to these two phenomena. Our results may be useful to develop potential treatments for related hearing diseases.

\section{Competing interests}

No conflicts of interest, financial or otherwise, are declared by the authors.

\section{Authors' contributions}

FW, LZ, YS, WG performed the experiments, LZ, FW analyzed experimental data, FW, BH and LL designed the project, LL and DH sponsored and supervised the experiments, LZ, FW and EMR wrote the paper, LZ and EMR made figures, LZ supervised the paper submission, editing, and revision. All authors read and approved the final manuscript.

\section{Acknowledgments}

We acknowledge the assistance of Dr. Alan R. Palmer, Brad Rose, Huizhan Liu, Yuqian Liu for research support. We also thank Allison Hallman, Yongjin Zhu, and William Roberts for their assistance.

\section{Grants}

This study was supported by National Natural Science Foundation of China (NSFC \#30872863), Michigan OU General Fund G110, and Ohio State University College of Medicine Start-up Fund 013000 (USA).

\section{Author details}

${ }^{1}$ Department of Otolaryngology, The First Affiliated Hospital of Chinese PLA General Hospital, Beijing 100048, China. ${ }^{2}$ Radiologic Sciences and Respiratory Therapy Division, School of Health and Rehabilitation Sciences, The Ohio State University College of Medicine, The Ohio State University Wexner Medical Center, Columbus, OH 43210, USA. ${ }^{3}$ Department of Biological Sciences, Molecular Physiology and Biophysics Laboratory, Oakland University, Rochester, Ml 48309, USA. ${ }^{\circ}$ Department of Biomedical Engineering, School of Medicine, Tsinghua University, Beijing 100084, China. ${ }^{5}$ Department of Otolaryngology-Head and Neck Surgery, Chinese PLA General Hospital, Beijing 100853, China. ${ }^{6}$ Department of Otolaryngology, Beijing Friendship Hospital, Capital Medical University, Beijing 100050, China.

Received: 1 October 2013 Accepted: 3 December 2013

Published: 9 December 2013

\section{References}

1. Bauer CA, Turner JG, Caspary DM, Myers KS, Brozoski TJ: Tinnitus and inferior colliculus activity in chinchillas related to three distinct patterns of cochlear trauma. J Neurosci Res 2008, 86:2564-2578.

2. Dong S, Mulders WH, Rodger J, Woo S, Robertson D: Acoustic trauma evokes hyperactivity and changes in gene expression in guinea-pig auditory brainstem. Eur J Neurosci 2010, 31:1616-1628.

3. Mulders WH, Robertson D: Hyperactivity in the auditory midbrain after acoustic trauma: dependence on cochlear activity. Neuroscience 2009, 164:733-746

4. Alkhatib A, Biebel UW, Smolders JW: Reduction of inhibition in the inferior colliculus after inner hair cell loss. Neuroreport 2006, 17:1493-1497.

5. Rajan R: Receptor organ damage causes loss of cortical surround inhibition without topographic map plasticity. Nat Neurosci 1998, 1:138-143.

6. Rajan R, Irvine DR, Wise LZ, Heil P: Effect of unilateral partial cochlear lesions in adult cats on the representation of lesioned and unlesioned cochleas in primary auditory cortex. J Comp Neurol 1993, 338:17-49.

7. Snyder RL, Bonham BH, Sinex DG: Acute changes in frequency responses of inferior colliculus central nucleus (ICC) neurons following progressively enlarged restricted spiral ganglion lesions. Hear Res 2008, 246:59-78.

8. Seki S, Eggermont JJ: Changes in spontaneous firing rate and neural synchrony in cat primary auditory cortex after localized tone-induced hearing loss. Hear Res 2003, 180:28-38.

9. Komiya $H$, Eggermont JJ: Spontaneous firing activity of cortical neurons in adult cats with reorganized tonotopic map following pure-tone trauma. Acta Otolaryngol 2000, 120:750-756.

10. Vogler DP, Robertson D, Mulders WH: Hyperactivity in the ventral cochlear nucleus after cochlear trauma. J Neurosci 2011, 31:6639-6645.

11. Mann ZF, Kelley MW: Development of tonotopy in the auditory periphery. Hear Res 2011, 276:2-15. 
12. Snyder RL, Sinex DG, McGee JD, Walsh EW: Acute spiral ganglion lesions change the tuning and tonotopic organization of cat inferior colliculus neurons. Hear Res 2000, 147:200-220.

13. Harrison RV, Stanton SG, Nagasawa A, Ibrahim D, Mount RJ: The effects of long-term cochlear hearing loss on the functional organization of central auditory pathways. J Otolaryngol 1993, 22:4-11.

14. Harrison RV, Stanton SG, Mount RJ: Effects of chronic cochlear damage on threshold and frequency tuning of neurons in Al auditory cortex. Acta Otolaryngol Suppl 1995, 519:30-35.

15. Seki S, Eggermont Jj: Changes in cat primary auditory cortex after minorto-moderate pure-tone induced hearing loss. Hear Res 2002, 173:172-186.

16. Izquierdo MA, Gutierrez-Conde PM, Merchan MA, Malmierca MS: Non-plastic reorganization of frequency coding in the inferior colliculus of the rat following noise-induced hearing loss. Neuroscience 2008, 154:355-369.

17. Salvi RJ, Wang J, Ding D: Auditory plasticity and hyperactivity following cochlear damage. Hear Res 2000, 147:261-274.

18. Schreiner $C E$, Langner G: Laminar fine structure of frequency organization in auditory midbrain. Nature 1997, 388:383-386.

19. Hernandez O, Espinosa N, Perez-Gonzalez D, Malmierca MS: The inferior colliculus of the rat: a quantitative analysis of monaural frequency response areas. Neuroscience 2005, 132:203-217.

20. Kaltenbach JA, Zacharek MA, Zhang J, Frederick S: Activity in the dorsal cochlear nucleus of hamsters previously tested for tinnitus following intense tone exposure. Neurosci Lett 2004, 355:121-125.

21. Kujawa SG, Liberman MC: Adding insult to injury: cochlear nerve degeneration after "temporary" noise-induced hearing loss. J Neurosci 2009, 29:14077-14085.

22. Norena AJ, Eggermont JJ: Enriched acoustic environment after noise trauma reduces hearing loss and prevents cortical map reorganization. J Neurosci 2005, 25:699-705.

23. Yang SM, Chen W, Guo WW, Jia S, Sun JH, Liu HZ, Young WY, He DZ: Regeneration of stereocilia of hair cells by forced Atoh1 expression in the adult mammalian cochlea. PloS one 2012, 7:e46355.

24. Rees A, Sarbaz A, Malmierca MS, Le Beau FE: Regularity of firing of neurons in the inferior colliculus. J Neurophysiol 1997, 77:2945-2965.

25. Liu LF, Palmer AR, Wallace MN: Phase-locked responses to pure tones in the inferior colliculus. J Neurophysio/ 2006, 95:1926-1935.

26. Hu BH, Guo W, Wang PY, Henderson D, Jiang SC: Intense noise-induced apoptosis in hair cells of guinea pig cochleae. Acta Otolaryngol 2000 120:19-24.

27. Cody AR, Robertson D: Variability of noise-induced damage in the guinea pig cochlea: electrophysiological and morphological correlates after strictly controlled exposures. Hear Res 1983, 9:55-70.

28. Wang $Y$, Hirose $K$, Liberman MC: Dynamics of noise-induced cellular injury and repair in the mouse cochlea. J Assoc Res Otolaryngol 2002, 3:248-268.

29. Mulders WH, Ding D, Salvi R, Robertson D: Relationship between auditory thresholds, central spontaneous activity, and hair cell loss after acoustic trauma. J Comp Neurol 2011, 519:2637-2647.

30. Liberman MC, Dodds LW: Single-neuron labeling and chronic cochlear pathology. III. Stereocilia damage and alterations of threshold tuning curves. Hear Res 1984, 16:55-74.

31. Terry AM, Jones DM, Davis BR, Slater R: Parametric studies of tinnitus masking and residual inhibition. Br J Audiol 1983, 17:245-256.

32. Adjamian P, Sereda M, Hall DA: The mechanisms of tinnitus: perspectives from human functional neuroimaging. Hear Res 2009, 253:15-31.

33. Dehmel S, Pradhan S, Koehler S, Bledsoe S, Shore S: Noise overexposure alters long-term somatosensory-auditory processing in the dorsal cochlear nucleus-possible basis for tinnitus-related hyperactivity? J Neurosci 2012, 32:1660-1671.

34. Brozoski TJ, Bauer CA: The effect of dorsal cochlear nucleus ablation on tinnitus in rats. Hear Res 2005, 206:227-236.

35. Fortune DS, Haynes DS, Hall JW: Tinnitus. Current evaluation and management. Med Clin North Am 1999, 83:153-162.

doi:10.1186/1423-0127-20-91

Cite this article as: Wang et al:: Tonotopic reorganization and spontaneous firing in inferior colliculus during both short and long recovery periods after noise overexposure. Journal of Biomedical Science $201320: 91$.

\section{Submit your next manuscript to BioMed Central and take full advantage of:}

- Convenient online submission

- Thorough peer review

- No space constraints or color figure charges

- Immediate publication on acceptance

- Inclusion in PubMed, CAS, Scopus and Google Scholar

- Research which is freely available for redistribution 